KANSAS JOURNAL of MEDICINE

\section{Urine Screening for Opioid and Illicit Drugs in the Total Joint Arthroplasty Population}

Matthew L. Vopat, M.D.', William G. Messamore, M.D., Ph.D.', Jesse J. Trent ${ }^{1}$, Ken E. Schmanke', Rosey Zackula, M.A.', Shang-You Yang, M.D., Ph.D., , , Tarun Bhargava, M.D., ${ }^{1,3}$

'University of Kansas School of Medicine-Wichita,

Department of Orthopaedics, Wichita, KS

${ }^{2}$ Wichita State University, Department of Biological Sciences, Wichita, KS

${ }^{3}$ Mid-America Orthopaedics, Wichita, KS

Received Sept. 10, 2019; Accepted for publication Dec. 13, 2019; Published online April 17, 2020

\section{ABSTRACT}

Introduction. Recent studies have shown an increase in postoperative orthopaedic complications associated with pre-operative opioid use. It is, therefore, important to know if patients use opioids before scheduled surgery. The purpose of this study was to determine if urine drug screening (UDS) is an effective screening tool for detecting opioid and illicit drug use prior to joint arthroplasty (JA) procedures.

Methods. This retrospective chart review was performed with IRB approval on 166 out of 172 consecutive patients in a communitybased practice. All the patients had a pre-operative UDS prior to primary or revision JA by a fellowship trained orthopaedic surgeon between March 2016 and April 2017. Patient demographics documented opioid and illicit drug use, co-morbid diagnosis, and UDS results were collected from clinical charts. Statistical analysis was conducted using Pearson Chi-square, Fisher's exact, McNemar test, and t-tests with IBM SPSS Statistics, ver. 23. Significant differences were $\mathrm{p}<0.05$.

Results. Sixty-four of 166 patients (38.6\%) tested positive for opioids. Among them, $55.0 \%$ (35/64) had no history of prescription opioid use. Significant differences were observed when comparing the test results of the UDS with the patient reported history of prescribed opioids $(\mathrm{p}=0.001)$.

Conclusion. With a significant number of patients testing positive for opioids without evidence of a previous prescription, UDS may be beneficial for initial risk assessment for patients undergoing JA procedures. Kans J Med 2020;13:71-76

\section{INTRODUCTION}

Alarming increases have occurred in the rate of drug overdose deaths involving synthetic opioids, such as tramadol, fentanyl, and its analogs. Data from the National Vital Statistics System (NVSS) reported that the age-adjusted rate of drug overdose has increased $88 \%$ per year from 2013 to 2016, and rose another $9.6 \%$ in 2017.1 This report also showed that trends for natural and semisynthetic opioids, such as oxycodone and hydrocodone, also have increased steadily. Similarly, the 2016 National Survey on Drug Use and Health (NSDUH) estimated 28.6 million Americans aged 12 years or older were current illicit drug users. ${ }^{2}$ These drugs included marijuana, cocaine (including crack), heroin, hallucinogens, inhalants, methamphetamines, and the misuse of prescription pain relievers such as tranquilizers, stimulants, and sedatives that were prescribed to someone else.

Prescription Opioid Use. A driver of this misuse is an overwhelming increase in physicians prescribing a long-term course of pain relievers, such as opioids, for chronic pain. ${ }^{3,4}$ One such condition is degenerative joint disease, or osteoarthritis, which is the leading cause of chronic pain and physical disability in older populations. ${ }^{5}$ Opioids, which are powerful pain-relieving substances, often are prescribed to patients with joint disease. Evidence indeed supports the effectiveness of opioids, showing an average 3-point decrease in pain as measured by a 10-point pain rating scale. ${ }^{5}$

However, studies have suggested an increase in post-operative orthopaedic complications associated with pre-operative opioid use.-11 Pre-operative drug users are at risk for longer hospital stays, have higher mortality rates, and more surgical complications, including postoperative infection, anemia, convulsions, osteomyelitis, and blood transfusion. ${ }^{12}$ More specifically, pre-operative opioid users have less favorable outcomes following total knee (TKA) and total hip arthroplasty (THA), ${ }^{3,13-17}$ besides the fact that they require more opioid refills, remain on opioids longer after surgery, and have an increased risk of chronic opioid use postoperatively. ${ }^{18-20}$ Evidence of common characteristics of those who use opioids include patients aged 35 to 49 years, taking five or more other medications, insured by Medicare or Medicaid, established versus new patients, treated by primary care versus specialty physicians, and in prescribing regions of the south or west. ${ }^{21}$

In recognition of this misuse, state-level prescription drug monitoring programs have been implemented in most states. Research to evaluate the effectiveness of these programs have been difficult because they vary by design and requirements. One study analyzed physician-reported data on prescribing opioids and found that these programs appeared to be unsuccessful when trying to reduce physicians prescribing opioids. ${ }^{22}$ It has been estimated that opioids are prescribed in 1 of 5 patients with non-cancer pain or pain-related diagnoses in an office-based setting.. ${ }^{23}$ This wide spread prescribing practice predisposes patients to chronic drug misuse and overdose related deaths. ${ }^{8,24}$

Illicit Drug Use. Seemingly, stricter enforcement of the prescription drug monitoring programs is merited and could lead to reduced drug misuse. However, this may lead to another issue in appropriate pain management: increased illicit drug use. ${ }^{25}$ Indeed, recent literature showed an increase in complications in the trauma population associated with illicit drug use. ${ }^{26}$ With ever increasing access to and use of illicit drugs in the United States, ${ }^{24}$ rates of drug misuse necessitates awareness and surveillance by all physicians. In elective orthopaedic procedures where the underlying goal is to improve the quality of the patient's life, it is important for orthopaedic physicians to have a thorough risk assessment of the patient to determine the most appropriate treatment course for his/her diagnosis. An accurate and up-to-date history and physical examination may be the best 
way to evaluate the patient's risks for surgery. However, due to negative perceptions of opioid and illicit drug use, patients may be reluctant to disclose this information to the physician.

Study Objective. To evaluate the extent to which patients disclose opioid and illicit drug use, we conducted urine drug screening (UDS) in our clinic for all patients who were scheduled for joint arthroplasty (JA). Our primary objective was to minimize complications and readmissions following $\mathrm{JA}$ in high-risk patients. The purpose of this study was to compare self-reported pre-operative drug use with UDS test results to determine if UDS would be an effective screening tool for detecting opioid and illicit drug use. We hypothesized that there would be a significantly high number of individuals in the JA population who do not reveal pre-operative drug use prior to surgery, thus putting themselves at undo risk of postoperative complications.

\section{METHODS}

Participants. A cross-sectional, retrospective chart review was performed on 172 consecutive patients in a community-based practice. Adult patients who had a pre-operative UDS prior to primary or revision JA were eligible to participate. Included in the subjects were patients scheduled for a JA between March 2016 and April 2017 by an adult reconstructive fellowship trained orthopaedic surgeon. JA was defined as primary or revision total hip arthroplasty (THA), total knee arthroplasty (TKA), patella femoral joint arthroplasty (PFJ), or uni-compartment knee arthroplasty (UKA).

This study was approved by the institutional review board (IRB). Each patient was told during the evaluation in the clinic to have a urine test for opioids and other associated illicit drugs prior to scheduling of the surgery. All patients used a private restroom with a member of the work staff outside the door in the senior author's clinic to collect their sample for the UDS. The UDS company used in this study was "Tru-Fit". All screens initially were examined by a strip in the clinic office, then confirmed off-site by the company using a gas chromatography. The UDS test cost the patient approximately sixty dollars, however, this varied with the type of insurance. At the time of presentation in clinic, the intake form that documented the patient's current prescribed drugs was initiated by the patient, then reviewed by a medical assistant with the patient to confirm the accuracy.

Measurements. Patient information was extracted from the charts, which included age, gender, body mass index (BMI), primary diagnosis, co-morbid diagnoses (including psychiatric disorders), smoking status, surgical date, history of medication list, type of insurance (Medicare, private, or workers' compensation), and JA procedure type. Data on comorbidities included diabetes, hypertension, hyperlipidemia, renal disease, major depression disorder, and general anxiety disorder. Pre-operative prescribed opioid use and UDS results also were collected.

Outcomes. The primary outcomes were the dichotomous UDStest result from four drug panels. The opiate/opioid drug panel included tests for 6-monoacetayl morphine, buprenorphine, codeine, EDDP, fentanyl, hydrocodone, hydromorphone, meperidine, methadone, morphine, naloxone, naltrexone, norbuprenorphine, norfentanyl, O-Desmethyl tramadol, oxycodone, oxymorphone, tapentadol, and tramadol. The remaining drug panels were categorized as illicit
KANSAS JOURNAL of MEDICINE URINE DRUG SCREENING IN TOTAL JOINT ARTHROPLASTY

continued.

drugs that included the amphetamine panel (amphetamine, MDA, MDEA, MDMA, methamphetamine, methylphenidate, and phentermine), the cannabinoid panel (THC, J-122_4-HYDROXYL and JWH-200), and the "other drug" panel (benzoylecgonine, dextromethorphan, mitragynine, PCP, and propoxyphene). For secondary outcomes, patients' pre-operative prescription medicine list was evaluated to determine if they were taking any pre-operative opioids. The number of patients taking pre-operative opioids was analyzed and compared to the patients that tested positive on UDS.

To eliminate false-positive results, the senior author offered a second drug screen to patients who tested positive for illicit drugs prior to cancelling their surgery.

Statistical Analyses. Data analysis was performed to determine the number of patients who had a positive urine toxicology for pre-operative opioid use or for illicit drugs, along with the number of patients who initially did not reveal the pre-operative drug use. Descriptive statistics included frequencies and percentages for categorical data. Continuous data were evaluated for normality and subsequently summarized with means and standard deviations. Groups were compared by positive or negative UDS results using the following two-tailed tests: Pearson Chi-square asymptotic and exact tests, McNemar test, and t-tests. Significant results were identified as those with $\mathrm{p}<0.05$. Analyses were conducted with IBM SPSS Statistics, ver. 23.

\section{RESULTS}

The analysis included 166 of the 172 patients; six patients were excluded because they underwent a procedure other than a JA. Data were missing on six participants for BMI, smoking, and insurance; two participants had missing data for comorbidities. The sample included 95 (57.2\%) females and 71 (42.8\%) males. The mean age was 64.5 years, with a standard deviation of 10.9 years. Many of the patients $(61.9 \%$; 99/160) were obese. Of the 166 patients screened with UDS, 64 (38.6\%) tested positive for opiate/opioids. Regarding the illicit drug panels, 7 (4.2\%) tested positive for Amphetamines, 6 (3.6\%) for Cannabinoids, and 2 (1.2\%) for other drugs (one participant tested positive for multiple drug panels within this category).

Table 1 summarizes participant characteristics by positive UDS results. Two participant characteristics significantly differed by UDS: over $44 \%$ (4l/92) of current/former smokers tested positive for opiate/opioids ( $\mathrm{p}=0.036)$, while almost $55 \%(18 / 33)$ diabetic patients tested positive $(\mathrm{p}=0.033)$. No other patient factors differed significantly by UDS results including sex, age, BMI, insurance type, type of scheduled arthroplasty, or other comorbidities. However, almost $40 \%$ of patients (34/92) scheduled for total knee arthroplasty tested positive for opiate/opioids; similarly, almost 44\% (2l/48) scheduled for total hip arthroplasty tested positive. (Note: because few patients tested positive for the three illicit drug panels, no other statistical tests were conducted for these drugs with only frequencies reported for these drug classes.) 


\section{KANSAS JOURNAL of MEDICINE}

URINE DRUG SCREENING INTOTAL JOINT

\section{ARTHROPLASTY}

\section{continued.}

Table 1. Participant characteristics by urine drug screen results.

\begin{tabular}{|c|c|c|c|c|c|c|c|}
\hline \multirow[b]{3}{*}{ Characteristics } & \multirow[b]{3}{*}{$\mathbf{N}(\%)$} & \multicolumn{6}{|c|}{ Positive Urine Drug Screen } \\
\hline & & \multicolumn{3}{|c|}{ Opiate/Opioid } & \multicolumn{3}{|c|}{ All Other } \\
\hline & & $\mathbf{N}$ & $\%$ & $\mathbf{p}$ & $\mathbf{N}$ & $\%$ & $\mathbf{p}$ \\
\hline Female & $95(57.2)$ & 37 & 57.8 & 0.904 & 9 & 64.3 & 0.577 \\
\hline Male & $71(42.8)$ & 27 & 42.2 & & 5 & 35.7 & \\
\hline Mean age (SD) & $166(100.0)$ & $63.5(11.8)$ & 0.342 & $59.6(10.9)$ & 0.081 & & \\
\hline BMI & & & & 0.991 & & & 0.389 \\
\hline Normal & $15(9.4)$ & 6 & 9.7 & & 1 & 7.1 & \\
\hline Overweight & $46(28.7)$ & 18 & 29.0 & & 2 & 14.3 & \\
\hline Obese & $99(61.9)$ & 38 & 61.3 & & 11 & 78.6 & \\
\hline Smoking status & & & & 0.036 & & & 0.213 \\
\hline Current & $31(19.4)$ & 18 & 29.0 & & 3 & 21.4 & \\
\hline Former & $61(38.1)$ & 23 & 37.1 & & 8 & 57.1 & \\
\hline Never & $68(42.5)$ & 21 & 33.9 & & 3 & 21.4 & \\
\hline Insurance type & & & & 0.982 & & & 0.314 \\
\hline Medicare/Medicaid & $89(55.6)$ & 34 & 55.7 & & 6 & 42.9 & \\
\hline Commercial & $71(44.4)$ & 27 & 44.3 & & 8 & 57.1 & \\
\hline Type of arthroplasty & & & & $0.536^{*}$ & & & $0.186^{*}$ \\
\hline Total knee: primary & $92(55.4)$ & 34 & 53.1 & & 6 & 42.9 & \\
\hline Total hip: primary & $48(28.9)$ & 21 & 32.8 & & 3 & 21.4 & \\
\hline Patella femoral joint & $11(6.6)$ & 3 & 4.7 & & 2 & 14.3 & \\
\hline Total knee: revision & $9(5.4)$ & 4 & 6.3 & & 2 & 14.3 & \\
\hline Total hip: revision & $3(1.8)$ & 2 & 3.1 & & 1 & 7.1 & \\
\hline $\begin{array}{l}\text { Uni-compartment } \\
\text { knee }\end{array}$ & $3(1.8)$ & 0 & 0.0 & & 0 & 0.0 & \\
\hline \multicolumn{8}{|c|}{ Co-morbidities (choose all that apply)** } \\
\hline Hypertension & $98(59.8)$ & 39 & 61.9 & 0.658 & 7 & 50.0 & 0.436 \\
\hline Diabetes & $33(20.1)$ & 18 & 28.6 & 0.033 & 3 & 21.4 & 0.899 \\
\hline Thyroid Disease & $28(17.1)$ & 14 & 22.2 & 0.166 & 1 & 7.1 & 0.302 \\
\hline Heart Disease & 27 (16.5) & 10 & 15.9 & 0.872 & 1 & 7.1 & 0.325 \\
\hline PMH of Cancer & $29(17.7)$ & 7 & 11.1 & 0.081 & 0 & 0.0 & 0.070 \\
\hline Rheumatoid Arthritis & 19 (11.6) & 6 & 9.5 & 0.515 & 3 & 21.4 & 0.229 \\
\hline
\end{tabular}

*Pearson Chi-square exact 2-sided test

** Other co-morbidities reported, but not listed in the table due to spare data: Atrial fibrillation $=7$, Chronic kidney disease $=8$, COPD $=8$, hypercholesterolemia

$=13$, psychiatric disorders $=10$. 
Positive UDS results were compared to self-reported history of prescribed opioids (Table 2). Results from the amphetamine panel showed seven patients tested positive, with four disclosing a history of prescribed opioids. For cannabinoids, six were positive, two of which disclosed a history of opioids. UDS results for the other drug panel showed two positive, with one self-reported opioid prescription. The opiate/opioid panel showed 38.6\% (64/166) tested positive, but 35 of 64 patients were using opioids without a prescription $(\mathrm{p}<0.01)$. Twenty-eight percent or 35 of 125 with no prescription for opioids test positive on UDS. Twenty-nine of 41 with a prescription of opioids tested positive on UDS. Thus, it appeared opioid use was significantly under-reported prior to surgery.

\section{DISCUSSION}

This study suggested that opioid use is relatively common in elderly patients who are evaluated for JA surgery in a community-based orthopaedic clinic. The results from this study also demonstrated that a significantly high portion of these patients have a positive UDS for opioids that failed to disclose this information on their initial preoperative examination. The prevalence of positive UDS for illicit drugs was higher than expected, especially for this relatively elderly population.

This study had limitations. The first limitation was the small population size from a single senior author's clinic with a limited number of cases in our study timeframe. The other limitation may come from

\section{KANSAS JOURNAL of MEDICINE}

\section{URINE DRUG SCREENING IN TOTAL JOINT}

ARTHROPLASTY

continued.

the relatively narrow detection time of UDS. For instance, oxycodone may not be detected if administered more than three days prior to testing, according to the Mayo Clinic Laboratories Guidance (https:// www.mayocliniclabs.com/test-info/drug-book/opiates.html). Also, this study did not consider the duration and dosage of opioid use, which Nguyen et. al. ${ }^{13}$ has shown to effect clinical outcomes.

However, recent studies such as Ben-Ari et al. ${ }^{3}$ found that 39.1\% of patients that underwent a TKA in the U.S. Veterans Affairs (VA) system were prescribed long-term opioids pre-operatively; $2.2 \%$ of these patients required a revision within a year. They concluded that long-term opioid use prior to TKA was associated with an increase in knee revisions. ${ }^{3}$ Similarly, Zywiel et al. ${ }^{17}$ found pre-operative opioid use prior to TKA was associated with diminished functional scores, increase risk for complications, and prolonged painful recovery. Sing et al. ${ }^{15}$ found an increased correlation between patients on pre-operative opioids prior to TKA and risk of complications within the first 90 days post-operatively. They further concluded that pre-operative opioid use should be disclosed as a risk for complications to patients and should be taken into consideration by the physicians prior to opioid management. All these studies justified that pre-operative

Table 2. Positive UDS test results for each drug and number of patients with history of prescribed opioids.

\begin{tabular}{|c|c|c|c|c|c|c|}
\hline \multirow[b]{3}{*}{ Positive Urine Drug Screen } & \multicolumn{6}{|c|}{ Self-Reported History of Prescribed Opioids } \\
\hline & \multicolumn{2}{|c|}{ Yes } & \multicolumn{2}{|c|}{ No } & \multicolumn{2}{|c|}{ Total } \\
\hline & $\mathrm{n}=41$ & $(24.7 \%)$ & $\mathrm{n}=125$ & $(75.3 \%)$ & $\mathrm{n}=166$ & $(100.0 \%)$ \\
\hline Amphetamines Panel & 4 & 9.8 & 3 & 2.4 & 7 & 4.2 \\
\hline Amphetamine & 2 & 4.9 & 2 & 1.6 & 4 & 2.4 \\
\hline Phentermine & 2 & 4.9 & 0 & 0.0 & 2 & 1.2 \\
\hline MDEA & 0 & 0.0 & 0 & 0.0 & 0 & 0.0 \\
\hline Methamphetamine & 0 & 0.0 & 2 & 1.6 & 2 & 1.2 \\
\hline Methylphenidate & 0 & 0.0 & 1 & 0.8 & 1 & 0.6 \\
\hline Cannabinoids Panel & 2 & 4.9 & 4 & 3.2 & 6 & 3.6 \\
\hline THC & 2 & 4.9 & 4 & 3.2 & 6 & 3.6 \\
\hline Opiate/Opioid Panel & 29 & 70.7 & 35 & 28.0 & 64 & 38.6 \\
\hline Oxymorphone & 13 & 31.7 & 4 & 3.2 & 17 & 10.2 \\
\hline Oxycodone & 12 & 29.3 & 3 & 2.4 & 15 & 9.0 \\
\hline O-Desmethyl tramadol & 10 & 24.4 & 14 & 11.2 & 24 & 14.5 \\
\hline Tramadol & 10 & 24.4 & 14 & 11.2 & 24 & 14.5 \\
\hline Hydrocodone & 7 & 17.1 & 17 & 13.6 & 24 & 14.5 \\
\hline Hydromorphone & 4 & 9.8 & 11 & 8.8 & 15 & 9.0 \\
\hline Morphine & 3 & 7.3 & 1 & 0.8 & 4 & 2.4 \\
\hline Codeine & 0 & 0.0 & 3 & 2.4 & 3 & 1.8 \\
\hline EDDP & 0 & 0.0 & 2 & 1.6 & 2 & 1.2 \\
\hline Fentanyl & 0 & 0.0 & 0 & 0.0 & 0 & 0.0 \\
\hline Methadone & 0 & 0.0 & 2 & 1.6 & 2 & 1.2 \\
\hline Other Drug Panel & 1 & 2.4 & 1 & 0.8 & 2 & 1.2 \\
\hline Dextromethorphan & 1 & 2.4 & 0 & 0.0 & 1 & 0.6 \\
\hline Benzoylecgonine & 0 & 0.0 & 1 & 0.8 & 1 & 0.6 \\
\hline
\end{tabular}


KANSAS JOURNAL of MEDICINE

\section{URINE DRUG SCREENING IN TOTAL JOINT}

ARTHROPLASTY

continued.

opioid use should be screened at the initial examination as part of a thorough risk analysis of the patient. Interestingly, these studies only looked at the reported prescriptions. The current study may illustrate that these patients need to be screened even further because patients have access to these medications beyond their current prescriptions.

Levy et al. ${ }^{27}$ evaluated the prevalence of illicit drug use in orthopaedic trauma patients. They found that patients tested positive for alcohol (25\%), cocaine (22\%), and marijuana (21\%). ${ }^{27}$ They further found that patients with a positive screen had more severe orthopaedic injuries and longer hospital stays. ${ }^{27}$ Similarly, Lank et al. ${ }^{26}$ also utilized the UDS to evaluate trauma patients over 55 years of age and found that patients who tested positive for marijuana and cocaine resulted in longer lengths of stay in the ICU compared to patients with negative UDS. This is in contrast to the current study, which had a more elderly patient population and patients who were evaluated for elective cases like JA. Our study found only $3.6 \%$ of patients tested positive for marijuana. However, this study was not performed where marijuana is legal. Furthermore, one could suspect that this prevalence would be higher in a state where marijuana has been legalized. With this increase in the number of states legalizing the use of marijuana, future studies need to evaluate how this will affect outcomes in patients who undergo elective orthopaedic cases such as JA procedures.

In this study, all histories of medication intake were clarified with the patients when the results of the urine drug screen returned. When excluding the patients testing positive for marijuana, $6 \%$ of patients in this study tested positive for other illicit drugs. This is clinically significant especially in a community-based practice such as this study was performed. The literature in how illicit drugs effect the outcomes with orthopaedic procedures is limited, especially in elective cases. ${ }^{26,27}$ However, the ability to evaluate illicit drugs such as cocaine, amphetamines, or heroin in elective JA procedures likely is limited for future research. It is more realistic for future studies to determine how canceling these cases may affect the overall complication rate.

With recent literature reporting an increase in post-operative complications with pre-opioid use,,$^{3,14-16}$ combined with the significantly high prevalence of pre-opioid and illicit drug use found in this elderly general population and patients not disclosing this information on their exam, it may be necessary to utilize the UDS for initial risk assessment for patients undergoing JA procedures. However, further studies need to evaluate how a positive UDS for pre-operative opioids effects patient's undergoing JA procedures complications post-operatively and function outcomes. This study found that access to these opioid drugs, other than what patients are prescribed, is prominent in the community-based orthopaedic clinic setting and further screening needs to be evaluated for pre-operative opioid use. Thus, UDS may be clinically helpful in assessing patient's risks preoperatively.

\section{CONCLUSION}

With a significant number of patients testing positive for opioids without evidence of a previous prescription, UDS may be beneficial for initial risk assessment for patients undergoing JA procedures.

\section{REFERENCES}

${ }^{1}$ Hedegaard H, Minino AM, Warner M. Drug overdose deaths in the United States, 1999-2017. NCHS Data Brief 2018; (329):1-8. PMID: 30500323.

2 Substance Abuse and Mental Health Services Administration. Key substance use and mental health indicators in the United States: Results from the 2016 National Survey on Drug Use and Health (HHS Publication No. SMA 17-5044, NSDUH Series H-52). 2017. Rockville, MD: Center for Behavioral Health Statistics and Quality, Substance Abuse and Mental Health Services Administration. Retrieved from https://www.samhsa.gov/data/.

${ }_{3}^{3}$ Ben-Ari A, Chansky H, Rozet I. Pre-operative opioid use is associated with early revision after total knee arthroplasty: A study of male patients treated in the veterans affairs system. J Bone Joint Surg Am 2017; 99(1):1-9. PMID: 28060227.

${ }^{4}$ Manchikanti L, Kaye AM, Knezevic NN, et al. Responsible, safe, and effective prescription of opioids for chronic non-cancer pain: American Society of Interventional Pain Physicians (ASIPP) guidelines. Pain Physician 2017; 20(2S):S3-S92. PMID: 28226332.

${ }^{5}$ da Costa BR, Nuesch E, Kasteler R, et al. Oral or transdermal opioids for osteoarthritis of the knee or hip. Cochrane Database Syst Rev 2014; (9):CD003115. PMID: 25229835.

${ }^{6}$ Chapman CR, Davis J, Donaldson GW, Naylor J, Winchester D. Postoperative pain trajectories in chronic pain patients undergoing surgery: The effects of chronic opioid pharmacotherapy on acute pain. J Pain 2011; 12(12):1240-1246. PMID: 22036517.

7 Cheah JW, Sing DC, McLaughlin D, Feeley BT, Ma CB, Zhang AL. The perioperative effects of chronic pre-operative opioid use on shoulder arthroplasty outcomes. J Shoulder Elbow Surg 2017; 26(11):1908-1914. PMID; 28735841.

${ }^{8}$ Devin CJ, Lee DS, Armaghani SJ, et al. Approach to pain management in chronic opioid users undergoing orthopaedic surgery. J Am Acad Orthop Surg 2014; 22(10):614-622. PMID: 25281256.

9 Lawrence JT, London N, Bohlman HH, Chin KR. Pre-operative narcotic use as a predictor of clinical outcome: Results following anterior cervical arthrodesis. Spine (Phila Pa 1976) 2008; 33(19):2074-2078. PMID: 18758363.

${ }^{10}$ Menendez ME, Ring D, Bateman BT. Pre-operative opioid misuse is associated with increased morbidity and mortality after elective orthopaedic surgery. Clin Orthop Relat Res 2015; 473(7):2402-2412. PMID: 25694266.

${ }^{11}$ Morris BJ, Sciascia AD, Jacobs CA, Edwards TB. Pre-operative opioid use associated with worse outcomes after anatomic shoulder arthroplasty. J Shoulder Elbow Surg 2016; 25(4):619-623. PMID: 26652698.

${ }_{12}$ Best MJ, Buller LT, Klika AK, Barsoum WK. Outcomes following primary total hip or knee arthroplasty in substance misusers. J Arthroplasty 2015; 30(7):1137-1141. PMID: 25765129.

${ }_{13}$ Nguyen LC, Sing DC, Bozic KJ. Pre-operative reduction of opioid use before total joint arthroplasty. J Arthroplasty 2016; 31(9 Suppl):282-287. PMID: 27105557.

${ }^{14}$ Pivec R, Issa K, Naziri Q, Kapadia BH, Bonutti PM, Mont MA. Opioid use prior to total hip arthroplasty leads to worse clinical outcomes. Int Orthop 2014; 38(6):1159-1165. PMID: 24573819.

${ }^{15}$ Sing DC, Barry JJ, Cheah JW, Vail TP, Hansen EN. Long-acting opioid use independently predicts perioperative complication in total joint arthroplasty. J Arthroplasty 2016; 31(9 Suppl):170-174 el. PMID: 27451080.

${ }_{16}$ Smith SR, Bido J, Collins JE, Yang H, Katz JN, Losina E. Impact of preoperative opioid use on total knee arthroplasty outcomes. J Bone Joint Surg Am 2017; 99(10):803-808. PMID: 28509820.

17 Zywiel MG, Stroh DA, Lee SY, Bonutti PM, Mont MA. Chronic opioid use prior to total knee arthroplasty. J Bone Joint Surg Am 2011; 93(21):19881993. PMID: 22048093.

${ }^{18}$ Hernandez NM, Parry JA, Mabry TM, Taunton MJ. Patients at risk: Preoperative opioid use affects opioid prescribing, refills, and outcomes after total knee arthroplasty. J Arthroplasty 2018; 33(7S):S142-S146. PMID: 29402712.

${ }^{19}$ Kim KY, Anoushiravani AA, Chen KK, Roof M, Long WJ, Schwarzkopf R. Pre-operative chronic opioid users in total knee arthroplasty-which patients persistently abuse opiates following surgery? J Arthroplasty 2018; 33(1):107-112. PMID: 28844770. 
${ }^{20}$ Zarling BJ, Yokhana SS, Herzog DT, Markel DC. Pre-operative and postoperative opiate use by the arthroplasty patient. J Arthroplasty 2016; 31(10):2081-2084. PMID: 27161903.

${ }^{21}$ Rasu RS, Knell ME. Determinants of opioid prescribing for nonmalignant chronic pain in us outpatient settings. Pain Med 2018; 19(3):524-532. PMID: 28340230.

${ }^{22}$ Lin HC, Wang Z, Boyd C, Simoni-Wastila L, Buu A. Associations between statewide prescription drug monitoring program (PDMP) requirement and physician patterns of prescribing opioid analgesics for patients with noncancer chronic pain. Addict Behav 2018; 76:348-354. PMID: 28898808.

${ }^{23}$ Daubresse M, Chang HY, Yu Y, et al. Ambulatory diagnosis and treatment of nonmalignant pain in the United States, 2000-2010. Med Care 2013; 5l(10):870-878. PMID: 24025657.

${ }^{24}$ Rudd RA, Seth P, David F, Scholl L. Increases in drug and opioid-involved overdose deaths - United States, 2010-2015. MMWR Morb Mortal Wkly Rep 2016; 65(50-51):1445-1452. PMID: 28033313.

${ }^{25}$ Beletsky L, Davis CS. Today's fentanyl crisis: Prohibition's Iron Law, revisited. Int J Drug Policy 2017; 46:156-159. PMID: 28735773.

${ }^{26}$ Lank PM, Crandall ML. Outcomes for older trauma patients in the emergency department screening positive for alcohol, cocaine, or marijuana use. Am J Drug Alcohol Abuse 2014; 40(2):118-124. PMID: 24588418.

${ }^{27}$ Levy RS, Hebert CK, Munn BG, Barrack RL. Drug and alcohol use in orthopedic trauma patients: A prospective study. J Orthop Trauma 1996; 10(1):2l-27. PMID: 8926551.

Keywords: opioid-related disorders, substance abuse detection, total joint replacement
KANSAS JOURNAL of MEDICINE

URINE DRUG SCREENING IN TOTAL JOINT

ARTHROPLASTY

continued. 